\section{El reconocimiento legal de la transexualidad en Chile mediante el procedimiento judicial de cambio de nombre. Un caso de complementariedad epistemológica entre medicina y derecho}

FERNANDO MUÑOZ LEÓNa

\section{Legal recognition of transsexuality in Chile through the judicial procedure for name change}

Do transsexual people in Chile have a right to have their gender identity or their sex reassignment legally recognized? The absence of any legislation on gender identity or transsexualism could lead us to believe that it is not the case. However, a quantitative review of decisions issued by Chilean courts during the last years on name-and sex-change requests filed by transsexual people reveals that most of these courts have accepted these requests. From the perspective of the well-being of transsexual people, this is a positive result. However, the fact that a few rejections exist reminds us of the need to enact an explicit legislation in this issue. Lastly, a qualitative analysis of those decisions suggests that the traditional reluctance of courts to interpret the law in a creative way has been overcome in these cases by the use of knowledge and discourses belonging to healthcare sciences. This is an example of an epistemological complementariness between medicine and law.

(Rev Med Chile 2015; 143: 1015-1019) lism.

Key words: Gender Identity; Names; Transgendered persons; Transsexua-

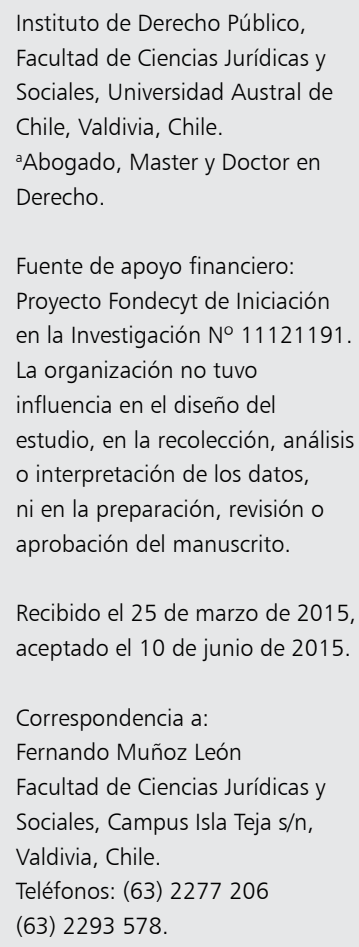

E ste trabajo forma parte de un proyecto de investigación que busca comprender las características de la discriminación en Chile y la manera en que el derecho puede contribuir a su fin. Así, se definiría por discriminación al heterogéneo cúmulo de desventajas que afectan a ciertas categorías de personas debido a que comparten un rasgo o cualidad que la mentalidad social prevaleciente identifica como relevante e interpreta como disvaliosa, a través de un proceso de categorización binaria que paralelamente identifica categorías opuestas de personas cuyo rasgo o cualidad compartida es interpretada como valiosa. En torno a cualidades 'adscritas' como la etnicidad o la orientación sexual se establecen asimetrías entre etnicidades privilegiadas (por ejemplo, los eurodescendientes) y etnicidades desventajadas (por ejemplo, los afrodescendientes), o bien entre orientaciones sexuales privilegiadas (por ejemplo, la heterosexualidad) y orientaciones sexuales desventajadas (por ejemplo, la homosexualidad). Los privilegios y desventajas asociados a cada situación discriminatoria adoptan las más diversas formas; van desde desiguales premios o castigos 
por iguales méritos y deméritos (piénsese en la brecha salarial entre hombres y mujeres) hasta la exclusión total de cierto tipo de instituciones (piénsese en la imposibilidad de las parejas del mismo sexo de contraer matrimonio en Chile), pasando por desiguales niveles de atención, por parte de las profesiones y las ciencias, respecto de las problemáticas de cada categoría de personas.

Entre las categorías de personas desventajadas en razón de su identidad adscriptiva se encuentra la transexualidad; esto es, la condición de quienes, debido a que han vivido experimentando un desacuerdo entre su sexo biológico y su sexo psicológico o disforia de género, deciden llevar a cabo un conjunto de procesos de reasignación de sexo. La disforia de género tensiona de manera significativa la percepción que quien la experimenta tiene de sí, y transforma a la imagen que proyecta de sí, en la medida en que no sea readecuada, en una fuente de problemas psicológicos para el sujeto. Para quien experimenta tales males, la adopción de medidas que establezcan una cierta armonía entre su subjetividad y su presentación en sociedad representa una necesidad fundamental. Pero la persona que ha llevado a cabo un proceso de reasignación de sexo, o persona transexual, a menudo debe enfrentarse a nuevos males; esto es, a la incomprensión y el rechazo de otros -incluyendo, en ocasiones, a las propias instituciones públicas- que insisten en adscribirle a la persona transexual su sexo 'original' como su sexo 'verdadero'.

En la actualidad, diversas aplicaciones prácticas de saberes y discursos posibilitan que quien experimenta disforia de género alcance mayores niveles de armonía y bienestar. Ello ocurre, por ejemplo, con varias ramas de las ciencias de la salud. La siquiatría contribuye diagnosticando el cuadro sintomático de la disforia de género; la intervención sicológica puede ayudar, tanto a quien la experimenta como a quienes le rodean, a comprender y aceptar la identidad de género sentida por dicho sujeto, así como su necesidad de readecuar su vida para alinearse con ella. La endocrinología provee de tratamientos hormonales que permiten proporcionarle al individuo una mayor sintonía con el género sentido, y además, en aquellos casos en los cuales el individuo lo desee, sirven de preparación para la realización de intervenciones quirúrgicas que completen el proceso de reasignación de sexo.

Por su parte, el derecho, en cuanto espacio institucional de administración profesional de las categorías de lo legal, juega un papel fundamental respecto del bienestar de la persona transexual. En sociedades como la chilena, el sexo en sí constituye una cualidad adscrita utilizada de manera generalizada para identificar a los individuos, y en torno al cual están estructuradas una gran cantidad de espacios sociales. Por ello, las transformaciones sicológicas, hormonales, anatómicas e indumentarias acometidas por una persona transexual a menudo no son suficientes. La persona transexual necesita interactuar cotidianamente con instituciones públicas y con terceros de una manera que no le estigmatice ni le visibilice de manera hostil. En el logro de ese objetivo, al derecho le corresponde la crucial responsabilidad de reconocer a través de sus categorías la reasignación de sexo experimentada por la persona transexual; el no reconocimiento de dicha reasignación expone cotidianamente a la persona transexual a malos entendidos, burlas e incomprensiones que perpetúan su padecimiento original, el sufrimiento producto de la disforia de género. Por supuesto, el reconocimiento legal de la nueva identidad no es solamente un asunto de importancia práctica, instrumental, para la persona transexual; aquel reconocimiento constituye en sí una importantísima forma de satisfacer su necesidad de recibir la aceptación de otros de su nueva identidad.

Chile, a diferencia de países como Argentina, carece de una ley sobre el reconocimiento del derecho a la identidad de género y de la transexualidad. Ello podría dar a entender que las personas que llevan a cabo procesos parciales o completos de reasignación de sexo carecen de un adecuado reconocimiento legal de su identidad. Sin embargo, el presente estudio explora la posibilidad de que las personas transexuales hagan uso del procedimiento de cambio de nombre contemplado en la Ley No 17.344 para lograr el reconocimiento de un nuevo nombre expresivo de una nueva identidad, así como para, más explícitamente aún, lograr el reconocimiento de su nuevo sexo.

En síntesis, este estudio examina si el procedimiento judicial de cambio de nombre proporciona a las personas transexuales en Chile un reconocimiento legal de su condición. Su objetivo primario consiste en responder a dicha interrogante. Su objetivo secundario consiste en determinar si dicho procedimiento judicial constituye un punto 
de encuentro entre las ciencias del cuidado de la salud y la ciencia jurídica.

\section{Material y Método}

El material analizado en este trabajo corresponde a sentencias de tribunales chilenos recaídas en solicitudes de cambio de nombre y de sexo presentadas por personas transexuales. A fin de dimensionar lo que dichas personas solicitan al tribunal, es conveniente tener presente que el artículo 1 de la Ley No 17.344, la cual entró en vigencia en 1970, autoriza a toda persona a solicitar "que se le autorice para cambiar sus nombres o apellidos" sólo cuando "unos u otros sean ridículos, risibles o la menoscaben moral o materialmente" o "cuando el solicitante haya sido conocido durante más de cinco años, por motivos plausibles, con nombres o apellidos, o ambos, diferentes de los propios". Si bien el concepto de menoscabo moral puede ser interpretado de manera amplia para incorporar o comprender la disforia de género $-y$ veremos que así ha ocurrido-, conspira contra dicha actitud hermenéutica el que nuestros tribunales se hayan caracterizado históricamente, como observa Hilbink $^{1}$, por "rehuir las interpretaciones independientes o innovadoras y refugiarse en fallos conservadores".

Las sentencias estudiadas fueron recopiladas a través del buscador de jurisprudencia proporcionado por el Poder Judicial en su página web. Si bien el buscador en cuestión no hace indicación de la fecha de inicio de sus registros, ni tampoco garantiza la identificación de la totalidad de las sentencias existentes sobre un tema, experiencias anteriores del investigador con el mismo servicio indican que aquel ofrece un servicio de alta fiabilidad respecto a las sentencias de, a lo menos, los últimos cinco años.

Dicho buscador permite obtener el texto íntegro de todas las sentencias alojadas en los servidores de dicha institución, a través del uso de criterios de búsqueda o 'voces'. En este caso, se emplearon las frases "cambio de sexo" y "Ley Zamudio". Las sentencias así identificadas fueron incorporadas a una tabla donde se registró el tribunal respectivo, el número identificador de la gestión o, en jerga judicial, su rol, la fecha de la sentencia, y el resultado de la misma, el cual se clasificó en tres categorías: "acoge", si el tribunal aceptó tanto la solicitud de cambio de nombre como de cambio de sexo; "acoge parcialmente", si el tribunal aceptó únicamente la solicitud de cambio de nombre y rechazó en cambio la solicitud de cambio de sexo; "rechaza", si el tribunal no aceptó ninguna de las dos solicitudes.

Los resultados obtenidos corresponden a información pública. Sin perjuicio de ello, el investigador se reserva las identidades de las personas que acudieron al procedimiento de cambio de nombre aquí estudiado.

\section{Resultados}

A través de la búsqueda anteriormente indicada se obtuvieron 86 solicitudes de cambio de sexo y de género. De ellas, 47 corresponden a personas transexuales femeninas (es decir, personas que solicitan que el tribunal reconozca su reasignación al sexo femenino) y 39 corresponden a personas transexuales masculinas (esto es, personas que solicitan que el tribunal reconozca su reasignación al sexo masculino). De estas solicitudes, 69 fueron acogidas, 6 fueron acogidas parcialmente, y 11 fueron rechazadas; dicho en términos porcentuales, $80,2 \%$ fueron acogidas, $7 \%$ fueron acogidas parcialmente, y $12,8 \%$ fueron rechazadas. De entre los 69 casos acogidos, 39 corresponden a personas transexuales femeninas, y 30 corresponden a personas transexuales masculinas. De entre los 6 casos acogidos parcialmente, 2 corresponden a personas transexuales femeninas, y 4 corresponden a personas transexuales masculinas. De entre los 11 casos rechazados, 6 corresponden a personas transexuales femeninas, y 5 corresponden a personas transexuales masculinas.

Las sentencias no siempre identifican si la persona solicitante se ha realizado una cirugía de reasignación de sexo, por lo que no constituyen una fuente confiable a efectos de determinar qué proporción de aquellas personas que acuden a este procedimiento han recurrido a tal cirugía. Debido a evidencia que proporcionan es circunstancial, no se intentará en esta etapa ofrecer datos al respecto.

Cabe observar que los resultados obtenidos guardan una cierta correspondencia con la información proporcionada por el Servicio de Registro Civil e Identificación a El Mercurio, aparecida con fecha 19 de enero de 2015 en dicho medio de comunicación, según la cual entre los años 2012 
y 2014, dicha institución recibió la instrucción, por parte de tribunales, de cambiar el sexo de 75 personas; 4 el 2012, 12 el 2013 y 22 el 2014. Los resultados aquí obtenidos ofrecen además un panorama más completo, debido a que incorporan un conjunto de tipos de casos más amplio que la información ofrecida por dicho Servicio, que sólo conoce de los casos acogidos, no de los casos de rechazo.

\section{Discusión}

El hecho de que los tribunales estén dispuestos a emplear de manera adaptativa o creativa el procedimiento judicial de cambio de nombre para reconocer legalmente el cambio de sexo es un hecho que un sector de la academia ya había observado hacía tiempo ${ }^{2}$. El presente estudio confirma este hecho a través de un estudio cuantitativo sistemático, cuyos hallazgos comprenden los últimos cuatro años. Aquí se ofrecerá una discusión de estos resultados que abordará dos aspectos: en qué medida este hallazgo es positivo desde el punto de vista de la dignidad y el bienestar de las personas transexuales; y qué nos dicen cualitativamente estos resultados sobre la relación entre ciencias del cuidado de la salud y saber jurídico.

En cuanto al bienestar y dignidad de las personas transexuales, los resultados encontrados son alentadores, pero no absolutamente positivos. La cifra de $80,2 \%$ de solicitudes acogidas es, por supuesto, alentadora, tomando en consideración las circunstancias institucionales y culturales en que se dan estas solicitudes, contexto al cual me referiré a continuación. Sin embargo, el que haya un total de 19,8\% de causas en las que no se acogió el cambio de sexo demuestra que existe el rechazo a la demanda transexual por reconocimiento en el procedimiento judicial de cambio de nombre; no es cierto que baste con la aplicación creativa del cuerpo legal en cuestión.

A fin de comprender qué nos dicen los resultados encontrados sobre la relación entre ciencias del cuidado de la salud y saber jurídico, es necesario recordar la afirmación formulada anteriormente: los tribunales chilenos, históricamente, se han caracterizado por su reticencia a interpretar las leyes de manera creativa. Hilbink ha argumentado que esta reticencia está anclada tanto en la trayectoria de las relaciones entre el Poder Judicial y el
Ejecutivo, caracterizada por la sumisión de aquel a este último, como en la configuración interna del Poder Judicial, caracterizada por la sumisión de los tribunales de primera instancia a las Cortes de Apelaciones y, en última instancia, a la Corte Suprema, sumisión que ha construido una jurisprudencia poco dada a la originalidad y a tomar riesgos $^{3}$. A esto se suma el que, como ha observado, entre otros, Squella ${ }^{4}$, nuestra cultura jurídica se caracteriza por la idea de que "los jueces, tanto en la interpretación como en la aplicación de las leyes, deben proceder, siempre e invariablemente, sin apartarse un punto de éstas, no siéndoles permitido llevar a cabo ninguna apreciación estimativa del texto de la ley ni de los efectos que pudieran derivarse de su aplicación estricta a un caso dado".

¿Qué explica, entonces, esta sucesión de casos de creatividad o, al menos, de flexibilidad judicial en relación con las personas transexuales? Una explicación posible consiste en la empatía judicial, entendiéndola como la capacidad de los jueces de imaginar el padecimiento de otros y la consiguiente apertura a modificar sus criterios interpretativos para acudir en su auxilio. Esta explicación permitiría enlazar estos casos con la conocida tendencia judicial, anterior a la ley de divorcio, a disolver legalmente los matrimonios a través del resquicio de declarar su nulidad por haber sido contraído el matrimonio en un domicilio distinto del correspondiente. Algunas de las sentencias examinadas parecen reflejar esta empatía judicial al insistir en la dimensión sicológica del padecimiento de la persona transexual.

Una segunda explicación consiste en la complementariedad epistemológica entre ciencias del cuidado de la salud y saber jurídico. Las solicitudes de cambio de sexo aquí analizadas se caracterizan por presentar una amplia documentación sobre los tratamientos hormonales y procedimientos quirúrgicos que han conducido a la reasignación de sexo. Pareciera ser que en este ámbito, el atrevimiento de los jueces a hacer a través de sus sentencias lo que el legislador no ha hecho a través de sus leyes se sustenta en un momento de apertura cognitiva a aplicaciones tecnológicas de saberes ampliamente validados, y que permiten someter la materia, en este caso la biología, al designio humano. Esta idea se ve respaldada por el hecho de que los pocos casos de aceptación parcial, esto es, de aceptación del cambio de nombre pero rechazo al cambio de sexo, ocurren fundamentalmente 
en casos en los cuales la persona transexual no ha 'completado' quirúrgicamente el proceso de reasignación sexual.

Los jueces son, en consecuencia, particularmente sensibles al lenguaje endocrinológico y quirúrgico. Quizás la complementariedad epistemológica aquí detectada no se verifica, entonces, entre el derecho y todas las ciencias del cuidado de la salud, sino más bien entre derecho y medicina. El problema es que ello refuerza la crítica del Centro de Derechos Humanos de la Universidad Diego Portales ${ }^{5}$, institución según la cual el que para lograr el cambio de sexo sea necesario someterse a una serie de operaciones y tratamientos médicos, como la extirpación de órganos y el tomar hormonas constituye una infracción de varios derechos humanos. Mal que mal, en la medida en que no haya un reconocimiento del derecho a cambiarse legalmente de nombre, de sexo, o de ambos, que sea exigible a todos los actores institucionales, la cirugía de reasignación de sexo, un procedimiento altamente invasivo que puede resultar en la pérdida de sensibilidad genital, se termina convirtiendo jurisprudencialmente en una especie de requisito o condición para el ejercicio del derecho a escoger la propia identidad de género.

La pregunta fundamental, entones, es si estamos dispuestos a reconocer a las personas el derecho a determinar autónomamente su identidad en materia de sexo/género. Si es así, entonces no solamente los procedimientos hormonales y qui- rúrgicos para realizar un proceso de reasignación de sexo debieran estar disponibles para todos, con independencia de la capacidad de pago; adicionalmente, la realización de dichos procedimientos no debiera constituir una exigencia para lograr el reconocimiento de la identidad de género experimentada por cada quien.

\section{Referencias}

1. Hilbink L. Jueces y política en democracia y dictadura: lecciones desde Chile. FLACSO. Ciudad de México. México. 2014. p. 23.

2. Centro de Derechos Humanos, Facultad de Derecho, Universidad Diego Portales. Diversidad sexual y derechos humanos. En: Informe Anual sobre Derechos $\mathrm{Hu}$ manos en Chile. Ediciones Universidad Diego Portales. Santiago. Chile. 2009. pp. 296-300.

3. Hilbink L. Jueces y política en democracia y dictadura: lecciones desde Chile. FLACSO. Ciudad de México. México. 2014.

4. Squella A. La cultura jurídica chilena. Corporación de Promoción Universitaria. Santiago. Chile. 1992. p. 42.

5. Centro de Derechos Humanos, Facultad de Derecho, Universidad Diego Portales. Informe sobre diversidad sexual: Las violaciones a los derechos humanos de las personas trans en Chile. En: Informe Anual sobre Derechos Humanos en Chile. Ediciones Universidad Diego Portales. Santiago. Chile. 2013; 322-5. 